\title{
Power Balance of Multi-Harmonic Components in Nonlinear Network
}

\author{
Binghua Huang, Xiaoyang He \\ Electrical Engineering School, Guangxi University, Nanning, China \\ Email: gxuhbh@163.com
}

Received 23 May 2014; revised 21 June 2014; accepted 19 July 2014

Copyright (C) 2014 by authors and Scientific Research Publishing Inc.

This work is licensed under the Creative Commons Attribution International License (CC BY). http://creativecommons.org/licenses/by/4.0/

c) (i) Open Access

\begin{abstract}
The main harmonic components in nonlinear differential equations can be solved by using the harmonic balance principle. The nonlinear coupling relation among various harmonics can be found by balance theorem of frequency domain. The superhet receiver circuits which are described by nonlinear differential equation of comprising even degree terms include three main harmonic components: the difference frequency and two signal frequencies. Based on the nonlinear coupling relation, taking superhet circuit as an example, this paper demonstrates that the every one of three main harmonics in networks must individually observe conservation of complex power. The power of difference frequency is from variable-frequency device. And total dissipative power of each harmonic is equal to zero. These conclusions can also be verified by the traditional harmonic analysis. The oscillation solutions which consist of the mixture of three main harmonics possess very long oscillation period, the spectral distribution are very tight, similar to evolution from doubling period leading to chaos. It can be illustrated that the chaos is sufficient or infinite extension of the oscillation period. In fact, the oscillation solutions plotted by numerical simulation all are certainly a periodic function of discrete spectrum. When phase portrait plotted hasn't finished one cycle, it is shown as aperiodic chaos.
\end{abstract}

\section{Keywords}

Frequency Domain, Complex Power, Difference Frequency, Nonlinear Coupling, Chaos, Harmonic, Oscillation

\section{Introduction}

Two types of mixing circuits containing three main harmonic components are introduced in this paper. The first type uses two signal sources with frequencies $\left(w_{1}, w_{2}\right)$ which are not the integer multiple of each other for mix- 
ing. The positive conductance with quadratic characteristic is used as a frequency conversion device to generate difference frequency $\left(w_{2}-w_{1}\right)$. There is only power-wasting positive resistance in the circuit, and self-excited oscillation cannot be produced. Three main harmonics are solved by frequency domain balance theorem and circuit law, and the complex power of each frequency component is individually conserved. The correctness of solving results is confirmed by traditional harmonic analysis method. The frequency components besides two signals will be produced in nonlinear circuit, for example there is no difference frequency excited source in circuit, but the power consumption of difference frequency component in the network can still be balanced. The power calculation in mixing circuit has important theoretical significance. It indicates that the balance theorem of frequency domain is applicable to not only odd term equation, but also even term equation. Circuit parameter design makes difference frequency component occupies a sufficient proportion in the overall mixing output, which has an important practical value.

The second type uses a nonlinear element with negative conductance and cubic characteristic. The circuit includes three main frequency components, the self-excited oscillation and two signal frequencies. The timedomain solution is expressed as the frequency-domain solution of Fourier series. There is no mathematical abstraction transformation formula for selecting appropriate harmonic term, so it can only rely on the physics background for establishing equation. The correct harmonic component can be sought by complex power balance theorem. This paper propels the application of theorem from single first harmonic to containing three main harmonics [1]-[8]. The mixing circuit structures of two types are identical, and only the various device parameters in the circuit and nonlinear characteristics are different. The interaction relation of nonlinear couplings of three main harmonics, and the calculation formulae of the first and second types are completely different.

\section{First Type Mixing Oscillation—Difference Frequency Is Main Harmonic Component}

\subsection{Three Main Harmonics Solved by Harmonic Analysis}

Symbols used in this paper are as follows, taking variable $u$ as example, $\bar{U}$ or the subscript $v$ represents phasor, $\bar{U}=u_{v}$. The subscript $m$ denotes amplitude, the $r$ denotes real part, the $x$ denotes imaginary part. For nonlinear branch, $i_{n}$ denotes instantaneous current, $i_{n 1}$ denotes the harmonic $w_{1}$ component of the $i_{n}$, while $i_{n 1 v}$ denotes the phasor of $i_{n 1}$, the $i_{n 1 m}$ denotes amplitude, $i_{n 1 r}$ denotes active component, $i_{n 1 x}$ denotes reactive component, $i_{n 2 v}$ denotes the phasor of $i_{n 2}$, the $i_{n 12 v}$ denotes the current phasor of difference frequency $w_{12}$. The electrical engineering symbol $j$ is used as the imaginary unit in this paper, for example, the phasor $u_{1 v}=u_{1 r}+j u_{1 x}$ in Example 1, and the phasor $u_{p 1 v}=U_{p 1 r}+j U_{p 1 x}$ in Example 2.

Example 1: The state equation of mixing model in Figure 1 is shown in (1), the scalar equation is shown in (2).

$$
\begin{gathered}
\dot{u}=-i_{N} / c-i_{L} / c+g_{F}\left(u_{F}-u\right) / c, \quad \dot{i}_{L}=u / L, i_{N}=b_{1} u+b_{2} u^{2}, \quad g_{N}=\mathrm{d} i_{N} / \mathrm{d} u=b_{1}+2 b_{2} u \\
\ddot{u}+h u+\left(g_{F}+g_{N}\right) \dot{u} / c=\dot{u}_{F} g_{F} / c \\
L=2500 / 213422881, \quad c=10^{-8}, h=1 / L c=w_{12}^{2}, g_{F}=1 / 100, \quad b_{1}=1 / 1000, b_{2}=2 / 1000 \\
w_{1}=3283221.01, w_{2}=6205021.01, w_{12}=w_{2}-w_{1}=2921800 \\
u_{F}=u_{F 1 r} \operatorname{sinzw_{1}}+u_{F 1 x} \operatorname{cosz}_{1}+u_{F 2 r} \operatorname{sinz} w_{2}+u_{F 2 x} \operatorname{coszw}_{2}, \\
u_{F 1 m}^{2}=u_{F 1 r}^{2}+u_{F 1 x}^{2}, u_{F 2 m}^{2}=u_{F 2 r}^{2}+u_{F 2 x}^{2}, \dot{u}_{F}=\mathrm{d} u_{F} / \mathrm{d} t \\
u_{123}=u_{1 r} \sin _{1} t+u_{1 x} \cos _{1} t+u_{2 r} \sin _{2} t+u_{2 x} \cos _{2} t+u_{12 r} \sin _{12} t+u_{12 x} \cos _{12} t, \quad z=t
\end{gathered}
$$

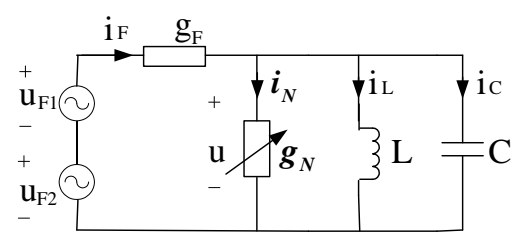

Figure 1. Mixing circuit. 
The variable $u$ contains a large number of frequency-doubling and combined-frequency components besides the two signal frequencies. In order to strengthen this available difference frequency component, the resonant frequency of LC circuit is designed to be equal to the difference frequency $w_{12}$ as shown in (2b). The excited source $u_{F}$ is shown in (2c). The three main harmonic components $u_{123}$ are set as shown in (3), where $w_{2}$ and $w_{1}$ denote two signal frequencies, and $w_{12}$ denote difference frequency. The $u_{1 r}, u_{1 x}, u_{2 r}, u_{2 x}, u_{12 r}, u_{12 x}$ are six undetermined coefficients. The LC circuit cannot produce self-oscillation while is used as selective filter circuit. Its task is to select difference frequency from the numerous frequency components of mixing. If the two excited sources are removed, namely let $u_{F}=0$ then $w_{12}$ cannot maintain self-excited. The difference frequency component relying on the two excitation support is not self-oscillation. It is commonly known as mid-frequency. The data of three main components are found by Program Tab1.nb, as listed in Table 1, where \% represents the percentage of difference frequency in all outputs, maxu represents the maximum total output.

\subsection{Result Verified by Power Balance Theorem}

The node method is used, and only one node voltage $u$ contains three harmonic components in circuit Figure 1. The $u$ is the variable of differential Equation (1), and the node voltage-phasor equation of three main harmonics is listed, as shown in (4).

$$
\begin{gathered}
\left(u_{F 1 v}-u_{1 v}\right) g_{F}=u_{1 v}\left(j w_{1} C+1 /\left(j w_{1} L\right)\right)+i_{n 1 v}, \quad\left(u_{F 2 v}-u_{2 v}\right) g_{F}=u_{2 v}\left(j w_{2} C+1 /\left(j w_{2} L\right)\right)+i_{n 2 v} \\
-u_{12 v} g_{F}=u_{12 v}\left(j w_{12} C+1 /\left(j w_{12} L\right)\right)+i_{n 12 v} \\
i_{n 1 v}=i_{1 r}+j i_{1 x}=b_{1}\left(u_{1 r}+j u_{1 x}\right)+b_{2} u_{12 x} u_{2 r}-b_{2} u_{12 r} u_{2 x}+j\left(b_{2} u_{12 r} u_{2 r}+b_{2} u_{12 x} u_{2 x}\right) \\
i_{n 2 v}=i_{2 r}+j i_{2 x}=b_{1}\left(u_{2 r}+j u_{2 x}\right)+b_{2} u_{12 x} u_{1 r}+b_{2} u_{12 r} u_{1 x}+j\left(b_{2} u_{12 x} u_{1 x}-b_{2} u_{12 r} u_{1 r}\right) \\
i_{n 12 v}=i_{12 r}+j i_{12 x}=b_{1}\left(u_{12 r}+j u_{12 x}\right)+b_{2} u_{1 x} u_{2 r}-b_{2} u_{1 r} u_{2 x}+j\left(b_{2} u_{1 r} u_{2 r}+b_{2} u_{1 x} u_{2 x}\right) \\
i_{n 1 v}=f_{1}\left(u_{1 v}, u_{2 v}, u_{12 v}\right), i_{n 2 v}=f_{2}\left(u_{1 v}, u_{2 v}, u_{12 v}\right), i_{n 12 v}=f_{12}\left(u_{1 v}, u_{2 v}, u_{12 v}\right)
\end{gathered}
$$

The (4) represents the current balance equations of three components according to node method denoted by phasor. If it is linear conductance $g_{\text {linear }}$, then $i_{n 1 v}=u_{1 v} g_{\text {linear }}$, and every harmonic component of node voltage $u$ can be independently solved by (4). There is no difference frequency component: $i_{n 12}=0$ and $u_{12}=0$ at this time. However, $g_{N}$ is the non-linear conductance, and $i_{n 1 v}$ is related to not only $u_{1 v}$ but also $u_{2 v}, u_{12 v}$. The coupling relation among three harmonic components is obtained by Program coupling.nb, as shown in (5). The current of a harmonic component includes the contributions of other harmonic voltage components. Therefore, the solution should be jointly found by (4) and (5). The real and imaginary parts of every phasor equation should be equal individually, so six equilibrium equations can be set up, six undetermined coefficients can be jointly solved as well. The data obtained by Program Power1.nb and Tab1.nb are consistent, as shown in Table 1.

Table 1. Main harmonic solution of Example 1.

(a)

$u F 1 r=6 ; u F 1 x=8 ; u F 2 r=4 ; u F 2 x=3 ; u F 1 m=1.45 ; \max u=8.92 ; \%=16.3 / 100 ;$

$u_{123}=-0.07 \cos [w 12 t]+2.76 \cos [w 1 t]-0.93 \cos [w 2 t]-1.45 \sin [w 12 t]+7.42 \sin [w 1 t]+0.4 \sin [w 2 t]$

(b)

$u F 1 r=6 ; u F 1 x=8 ; u F 2 r=6.2 ; u F 2 x=5 ; u F 1 m=2.38 ; \max u=9.81 ; \%=24.3 / 100$;

$u_{123}=-0.19 \cos [w 12 t]+2.68 \cos [w 1 t]-1.47 \cos [w 2 t]-2.38 \sin [w 12 t]+7.77 \sin [w 1 t]+0.638 \sin [w 2 t]$

(c)

$u F 1 r=6 ; u F 1 x=8 ; u F 2 r=8 ; u F 2 x=6 ; u F 1 m=3.05 ; \max u=10.47 ; \%=29 / 100$

$u_{123}=-0.09 \cos [w 12 t]+2.58 \cos [w 1 t]-1.86 \cos [w 2 t]-3.05 \sin [w 12 t]+8.1 \sin [w 1 t]+0.65 \sin [w 2 t]$

(d)

$u F 1 r=6 ; u F 1 x=8 ; u F 2 r=12 ; u F 2 x=9 ; u F 1 m=4.88 ; \max u=12.3 ; \%=39.7 / 100$;

$u_{123}=-0.0038 \cos [w 12 t]+2.11 \cos [w 1 t]-2.77 \cos [w 2 t]-4.88 \sin [w 12 t]+9.22 \sin [w 1 t]+0.63 \sin [w 2 t]$

(e)

$u F 1 r=7 ; u F 1 x=10 ; u F 2 r=12 ; u F 2 x=9 ; u F 1 m=5.13 ; \max u=13.6 ; \%=37.7 / 100$;

$u_{123}=1.3 \cos [w 12 t]+3.18 \cos [w 1 t]-2.55 \cos [w 2 t]-4.97 \sin [w 12 t]+10.6 \sin [w 1 t]+0.088 \sin [w 2 t]$ 
Finally, every harmonic power sum given by the program is identically equal to zero. It is verified that the power profit and loss of each harmonic component cannot be mutually replenished and will be individually conserved. The (5) can be represented as the general form shown in (6).

It should be pointed out that there is no difference frequency excited source in the circuit Figure 1. The power consumed by the difference frequency component in the positive conductance $g_{F}$ is supplied by the nonlinear element $g_{N}$ of variable-frequency. Taking the Table $1(\mathrm{e})$ as example, the $p_{n 12}$ which represents the active power consumed by branch $g_{N}$ is negative as shown in (7). The $p_{g F 12}$ which represents the active power consumed by branch $g_{F}$ is positive, as shown in (8). The active power consumed by $g_{N}$ and $g_{F}$ is completely balanced. The currents of branches $\mathrm{L}$ and $\mathrm{C}$ from difference frequency component are equal in size and opposite in direction. The consumed reactive power totals zero, as shown in (9).

$$
\begin{gathered}
S_{n 12}=p_{n 12}+j Q_{n 12}=u_{12 v} * \hat{i}_{n 12 v}=-0.2636345881+j_{0}, \quad \hat{i}_{n 12 v}=\text { Conjugate }\left[i_{n 12 v}\right], Q_{n 12}=0 \\
S_{g F 12}=p_{g F 12}+Q_{g F 12}=u_{g F 12 v} * \hat{i}_{F 12 v}=0.263634588+j_{0}, \hat{i}_{F 12 v}=\text { Congugate }\left[i_{F 12 v}\right], Q_{g F 12}=0 \\
\bar{U}_{12}\left[j w_{12} C+1 /\left(j w_{12} L\right)\right]=j \bar{U}_{12}\left[j w_{12} C+1 /\left(j w_{12} L\right)\right]=0
\end{gathered}
$$

The operation results of the Program Power1.nb show that the nonlinear element $g_{N}$ only consume active power, three main harmonics do not consume reactive power $\left(Q_{n 12}, Q_{n 1}, Q_{n 2}\right)=(0,0,0)$.

\subsection{Frequency Conversion and Power Conservation}

After frequency conversion, the conservation theorem still is valid. Taking the transformer as an example shows the principle of variable-frequency. For example, high-voltage $(6.3 \mathrm{kV})$ generator sends power to low-voltage (380/220 volts) users through a step-down transformer. Moreover, the power sent by generator and the power received by transformer on high-voltage side (including internal transformer consumption and power transformed into low voltage side) should be balanced. The power sent by low voltage side should be equal to that consumed by user. The power sum of two voltage levels should be individually balanced in this electric network. Transformer is a power source of the user on the low voltage side, but transformer itself does not produce power. It only plays the role of transforming high-voltage power into low-voltage power.

Industrial users of containing frequency conversion device use other frequencies to consume power. However, the power company sends $50 \mathrm{~Hz}$ frequency power to users, and then frequency conversion device will receive $50 \mathrm{~Hz}$ power. Therefore, the power system should maintain the complex power balance of $50 \mathrm{~Hz}$ frequency component. The power of frequency component after conversion should also maintain its power balance.

Two excited sources send power to the network containing frequency conversion element. Moreover, power sent by two excited sources using excited frequency and the power received by network (including the power consumption of internal network and the power transformed into difference frequency) should be balanced. The difference frequency power sent by frequency conversion element should also be equal to the power consumed by positive resistance. The power sum of three frequency components should individually be balanced in this electric network.

\section{Second Type Mixing Oscillation}

\subsection{Three Main Harmonic Solved by Harmonic Analysis}

$$
\begin{gathered}
g_{F}=24 \times 10^{-5}, \quad c=10^{-6}, \quad L=1.5625, \quad h_{a}=1 /(L c), \quad \omega_{1}=1260, \quad \omega_{2}=1860, \quad a_{1}=72 \times 10^{-5}, \quad a_{3}=16 \times 10^{-7} \\
\dot{u}=-i_{N} / c-i_{L} / c+g_{F}\left(u_{F}-u\right) / c, \quad \dot{i}_{L}=u / L, \quad \dot{z}=1, \quad i_{N}=-a_{1} u+a_{3} u^{3}, \quad g_{N}=\mathrm{d} i_{N} / \mathrm{d} u, \quad i_{N}=g_{N} \dot{u} \\
\ddot{u}+h_{a} u+\left(g_{F}+g_{N}\right) \dot{u} / c=\dot{u}_{F} g_{F} / c \\
u_{F}=u_{F 1}+u_{F 2}=u_{F 1 r} \sin z \omega_{1}+u_{F 1 x} \cos z \omega_{1}+u_{F 2 r} \sin z \omega_{2}+u_{F 2 x} \cos z \omega_{2}, \quad z=t \\
h_{a}=\omega_{h}^{2}, \quad U_{h m 0}^{2}=4\left(a_{1}-g_{F}\right) /\left(3 a_{3}\right), \quad\left(\omega_{h}, U_{h m 0}\right)=(800,20), \quad u_{h}=U_{h m 0} \sin \left(\omega_{h} t+\theta\right) \\
u_{123}=U_{p 1 r} \sin \omega_{1} t+U_{p 1 x} \cos \omega_{1} t+U_{p 2 r} \sin \omega_{2} t+U_{p 2 x} \cos \omega_{2} t+U_{h r} \sin \omega_{h} t+U_{h x} \cos \omega_{h} t
\end{gathered}
$$




$$
U_{h m}^{2}=U_{h r}^{2}+U_{h x}^{2}, \quad U_{p 1 m}^{2}=U_{p 1 r}^{2}+U_{p 1 x}^{2}, \quad U_{p 2 m}^{2}=U_{p 2 r}^{2}+U_{p 2 x}^{2}
$$

Example 2: Circuit is shown in Figure 1. Each element parameter are shown in (10), they is different from Example 1. The state equation is shown in (11), scalar equation is shown in (12). The comparison between state (11) and (1) shows that the two equations are identical in form, the comparison between scalar (12a) and (2a) shows that the two equations are also identical in form, and only their parameters and nonlinearities in the formulae are different, where $\omega_{1} \neq w_{1}, \omega_{2} \neq w_{2}, \omega_{h} \neq w_{12}$.

Let $u_{F}=0$, the self-oscillation $\omega_{h}$ still exists due to $g_{N}$ has negative conductance term. The $\left(\omega_{h}, U_{h m 0}\right)$ of the harmonic $u_{h}$ of self-oscillation are solved by Program Self.nb, as shown in (13), where $U_{h m 0}$ denotes amplitude of self-oscillation when $u_{F}=0$. The initial phase angle $\theta$ is arbitrary, it means the harmonic solution has no determined $\theta$. The self-oscillation of nonlinear circuit tends to a stationary limit cycle after the transient process. The circuit entering steady state certainly possess a transient time $t_{h}$ later from beginning. The initial phase angle $\theta$ of first movement of entering steady state cannot be determined. The $\theta$ and $t_{h}$ depend on different initial conditions, they cannot be sought. The stationary oscillation solution can be sought by harmonic analysis and power equilibrium theorem. The transient process is not an oscillation solution before entering limit cycle, which does not consist of harmonics and thus cannot be found by the harmonic analysis.

Let $u_{F} \neq 0$, three main harmonics containing self-oscillation are obtained by Program uhp.nb, as shown in (14). The comparison between (14) and (3) shows that the two formulae are also identical in form, and only their coefficients in the formulae are different, where

$$
\left(u_{1 r}, u_{1 x}, u_{2 r}, u_{2 x}, u_{12 r}, u_{12 x}, u_{12 m}\right) \neq\left(U_{p 1 r}, U_{p 1 x}, U_{p 2 r}, U_{p 2 x}, U_{h r}, U_{h x}, U_{h m}\right) \text {. }
$$

The solutions of self-excited and forced oscillation can be sought by superposition in linear equation. The coupling solution of self-excited and forced components should be jointly sought by Program uhp.nb.

Note that the self-excited oscillation is inhibited and disappears due to nonlinear coupling interaction when the combination strength of the $u_{F}$ is strong enough [9]-[12]. At this time, setting the $U_{h m}^{2}=U_{h r}^{2}+U_{h x}^{2}=0$ in (14), and two main harmonics containing only forced oscillation are obtained by Program onlyup.nb.

The results solved by Programs uhp.nb and onlyup.nb are listed in Table 2.

$$
\text { Table 2. Main harmonic solution of Example 2, upmupm }=2\left(U_{p 1 m}^{2}+U_{p 2 m}^{2}\right) \text {. }
$$

Self-oscillation existence = SOE, namely the operation result from program uhp.nb. the (uhx, uhr) is multi-solution. Self-oscillation disappearance $=\mathrm{SOD}$, namely the operation result from program onlyup.nb. $u h x \cos [800 t]+u h r \sin [800 t]=u h m \sin [800 t+\theta], u h m^{2}=u h x^{2}+u h r^{2}$ the $\theta$ is arbitrary

(a)

$u F 1 r=12 ; u F 1 x=16 ; u F 2 r=20 ; u F 2 x=15 ; u F 1 m=20 ; u F 2 m=25 ; u h m=17.4 ; \quad$ upmupm =98.78; SOE $u_{h p}=13.5 \cos [800 t]-1.4 \cos [1260 t]-2.5 \cos [1860 t]+10.85 \sin [800 t]+5.7 \sin [1260 t]+2.94 \sin [1860 t]$

(b) $u F 1 r=24 ; u F 1 x=32 ; u F 2 r=20 ; u F 2 x=15 ; u F 1 m=40 ; u F 2 m=25 ; u h m=7.21 ; \quad$ upmupm = 348.0; SOE $u_{h p}=5.8 \cos [800 t]-9.13 \cos [1260 t]-3.1 \cos [1860 t]+4.25 \sin [800 t]+8.66 \sin [1260 t]+2.46 \sin [1860 t]$

(e) $u F 1 r=36 ; u F 1 x=48 ; u F 2 r=40 ; u F 2 x=30 ; u F 1 m=60 ; u F 2 m=50 ; \quad$ upmupm $=837>400$ $u_{h p}=-9.5 \cos [1260 t]-4.5 \cos [1860 t]+16.5 \sin [1260 t]+6.1 \sin [1860 t] ;$ SOD $u F 1 r=12 ; u F 1 x=16 ; u F 2 r=72 ; u F 2 x=54 ; u F 1 m=20 ; u F 2 m=90 ; u p m u p m=484>400$

(f) $u_{h p}=-3.46 \cos [1260 t]-12.1 \cos [1860 t]+5.34 \sin [1260 t]+7.4 \sin [1860 t]$; SOD

(g) $\begin{aligned} & u F 1 r=0 ; u F 1 x=0 ; u F 2 r=96 ; u F 2 x=72 ; \\ & u_{p}=-15.54 \cos [1860 t]+10.914 \sin [1860 t] ; \text { SOD }\end{aligned}$ 
It should be noted that the initial value is not introduced, but the self-oscillation amplitude $u h m=U_{h m}$ solved by Program uhp.nb has determined. However, the $U_{h m} \neq U_{h m 0}$, the $\theta$ cannot be specifically determined. Therefore, the $(u h r, u h x)=\left(U_{h r}, U_{h x}\right)$ is multi-solution. Above is shown in Programs uhp.nb and Table 2.

$$
\begin{gathered}
U_{\text {eqhm }}^{2}=U_{h m 0}^{2}=U_{h m}^{2}+2\left(U_{p 1 m}^{2}+U_{p 2 m}^{2}\right), \quad U_{\text {eqp } 1 m}^{2}=U_{p 1 m}^{2}+2\left(U_{h m}^{2}+U_{p 2 m}^{2}\right), \quad U_{\text {eqp } 2 m}^{2}=U_{p 2 m}^{2}+2\left(U_{h m}^{2}+U_{p 1 m}^{2}\right) \\
g_{\text {eqh }}=-a_{1}+3 a_{3} U_{\text {eqhm }}^{2} / 4, \quad g_{\text {eqp } 1}=-a_{1}+3 a_{3} U_{\text {eqp } 1 m}^{2} / 4, \quad g_{\text {eqp } 2}=-a_{1}+3 a_{3} U_{\text {eqp } 2 m}^{2} / 4 \\
I_{h m}=U_{h m} g_{\text {eqh }}, \quad I_{p 1 m}=U_{p 1 m} g_{\text {eqp } 1}, \quad I_{p 2 m}=U_{p 2 m} g_{\text {eqp } 2}
\end{gathered}
$$

The mutual coupling relationship among three main harmonic components is shown in (15)-(17). One of harmonic current includes the contributions of the other harmonic voltage components. The concepts and definitions of equivalent voltage amplitude $U_{\text {eqm }}$ can be formed by (15), the equivalent conductance $g_{\text {eq }}$ can be defined by (16), and The current $I_{h m}$ of self-oscillation in (17) is equal to the product of self-oscillation voltage and equivalent conductance $g_{\text {eqh }}$, where $g_{\text {eqh }}$ contains the contributions of other harmonic voltage.

The initial condition is not introduced by the two examples in this paper, thus merely steady state solutions can be found. However, the distinction between difference frequency and self-oscillation components can be shown by the (5) and (17). In the (5), $i_{n 12 v}$ and $u_{12 v}$ are from the conversion of the excited source, they disappear when $u_{F}=0$, therefore the $u_{12 v}=u_{12 r}+j u_{12 x}$ is forced component, is unique determination. But in (17), the $I_{h m}$ and $U_{h m}$ are self-oscillation component, their power are not from the excited source, they still exist when $u_{F}=0$, thus the $U_{h m}$ is unique determination, while the $\left(U_{h r}, U_{h x}\right)$ is multi-solution.

\subsection{Using Phasor Method and Power Balance Theorem}

Using phasor method and frequency domain balance theorem (including frequency domain KCL and KVL as well as power equilibrium theorem) can obtain the conclusions which are consistent with those of harmonic analysis.

Firstly, let $u_{F}=0$, the $\left(\omega_{h}, U_{h m 0}\right)$ can be obtained by fundamental wave equilibrium principle, as shown in (13). If self-oscillation component exists, then mutual relationship among $U_{h m}$ and four unknown coefficients $\left(U_{p 1 r}, U_{p 1 x}, U_{p 2 r}, U_{p 2 x}\right)$ is shown in (15) and (18).

$$
\begin{gathered}
U_{h m}^{2}=U_{h m 0}^{2}-2\left(U_{p 1 r}^{2}+U_{p 1 x}^{2}+U_{p 2 r}^{2}+U_{p 2 x}^{2}\right), \quad U_{h m 0}=U_{e q h m} \\
\left(u_{F 1 v}-u_{p 1 v}\right) g_{F}=u_{p 1 v}\left[\left(j w_{1} C+1 /\left(j w_{1} L\right)\right)+g_{e q p 1}\right], \quad u_{p 1 v}=U_{p 1 r}+j U_{p 1 x}, U_{p 1 m}^{2}=U_{p 1 r}^{2}+U_{p 1 x}^{2} \\
\left(u_{F 2 v}-u_{p 2 v}\right) g_{F}=u_{p 2 v}\left[\left(j w_{2} C+1 /\left(j w_{2} L\right)\right)+g_{e q p 2}\right], \quad u_{p 2 v}=U_{p 2 r}+j U_{p 2 x}, U_{p 2 m}^{2}=U_{p 2 r}^{2}+U_{p 2 x}^{2}
\end{gathered}
$$

Secondly, if $u_{F} \neq 0$, the current equilibrium equation of two forced components is set up by using node method denoted by phasor, as shown in (19). The $g_{\text {eqp } 1}$ and $g_{\text {eqp } 2}$ in the above formulae are related to the voltage amplitudes of three harmonics, as shown in (16). The real and imaginary parts must individually be equal for each phasor equation, so four equilibrium equations can be set up to solve jointly four undetermined coefficients. The solutions from Programs haveself.nb are consistent with Program uhp.nb.

The equivalent conductance $g_{\text {eqh }}$ for $g_{N}$ is only relevant with the equivalent voltage amplitude $U_{\text {eqhm }}=U_{h m 0}$ as shown in(16), the $\left(\omega_{h}, U_{h m 0}\right)$ only depends on the specific parameters of network; and is irrelevant to the excited $u_{F}$. The $g_{e q h}$ independent from initial phase angle $\theta$, while the $\theta$ depends on the initial conditions, Therefore the $g_{\text {eqh }}$ independent from initial conditions.

If $u_{F} \neq 0$, and the self-oscillation does not exist when the $u_{F}$ is strong enough. The solutions of the (12) only contain two forced component, they can be obtained by Program nonself.nb. They are consistent with the solutions by Program onlyup.nb. All results obtained by phasor method and by harmonic balance method all are consistent, as shown in Table 2.

\subsection{Existence of Self-Oscillation Depending on Power Balance Conditions}

$U_{h m}^{2}=U_{h m 0}^{2}-2\left(U_{p 1 m}^{2}+U_{p 2 m}^{2}\right), \Delta=\operatorname{sgn}\left[U_{h m}^{2}\right]=\operatorname{sgn}\left[U_{h m 0}^{2}-2\left(U_{p 1 m}^{2}+U_{p 2 m}^{2}\right)\right]$, upmupm $=2\left(U_{p 1 m}^{2}+U_{p 2 m}^{2}\right)$

If $u_{F} \neq 0$, the (20) can be obtained from (18), then $U_{h m 0}^{2}>U_{h m}^{2}>0$ when self-oscillation exists. If $U_{h m}^{2}<0$ 
is obtained, it indicates that the self-oscillation has disappeared for stronger $u_{F}$. Whether self-oscillation exists can be determined by using the (20). Self-oscillation exists when $\Delta=1$; self-oscillation disappears when $\Delta=-1$.

\subsection{Incongruous with Power Balance Conditions Results in Wrong Conclusion}

The first case suppose harmonic solutions contain self-oscillation component, the wrong result will be obtained when external excited source is larger such as $U_{F 1 m}=60, U_{F 2 m}=50$. We obtain the imaginary $U_{h m}=4.33 j$. The $U_{h m}^{2}<0$ is found by Program uhpwrong.nb, so it can be determined by (20) that the self-oscillation has disappeared. The harmonic solutions should be reset, and self-oscillation component is removed. The correct results in Table 2(e) can be obtained by Program onlyup/E.nb.

The second case suppose harmonic solution exclude self-oscillation component, the wrong result will also be obtained when external excited source is small such as $U_{F 1 m}=40, U_{F 2 m}=25$. It can be determined that selfoscillation exists according to (20). we can obtain the (21) from Program upwrong.nb.

$$
\text { up }=-10 \cos [1260 t]-3.33 \cos [1860 t]+6.6 \sin [1260 t]+2.12 \sin [1860 t], \text { upmupm }=320<U_{h m 0}^{2}=400
$$

The harmonic solution should be reset as including self-oscillation, and the correct results in Table 2(b) can be obtained by Program uhp/B.nb. The solution set by harmonic analysis is a trial solution. It has very important significance that the containing harmonics component are estimated from the physical background.

In the first case, the results satisfying the equation equilibrium are all complex roots when $u_{F}$ is larger and errors obviously exist in them. For the second case, set harmonic component only contains two main harmonics, and real solution can also be obtained, as shown in (21). In order to find such hidden error, it is necessary to understand the physical background containing self-excited oscillation. Whether this solution is correct should be verified according to the (20) and (21).

\section{Two Types of Oscillation Characters}

\subsection{Phase Portrait of First Type Mixing Oscillation}

There is no negative conductance to release energy, so there is no self-oscillation component. Two excitation signals are finite energy source, and their mixing oscillation outputs are bounded. Periodic oscillation or chaos may be formed. The $u-\dot{u}$ plane phase portraits of overall output of mixing oscillation are drawn by Simulink. Taking Table 1(e) as an example, phase portraits are shown in Figure 2, where Figure 2(a) takes $100 \%$ of the phase points, and Figure 2(b) takes the last $5 \%$ of the phase points. They cannot show intuitively periodic or chaotic state.

In order to judge phase portrait property, the (1) and (2) are converted into three-dimensional state equation as shown in (22a), the phase portrait behavior is verified by Lyapunov Exponent (LE). Jacobi matrix is shown in (22b), where $\dot{u}_{F}$ denoted by (2c).

$$
\begin{gathered}
\dot{u}=-\left(b_{1}+g_{F}+b_{2} u\right) u / c-i_{L} / c+g_{F} u_{F} / c, \quad i_{L}=u / L, \quad \dot{z}=1, \quad z=t \\
J=\left[\begin{array}{ccc}
-\left(b_{1}+g_{F}+2 b_{2} u\right) / c & -1 / c & g_{F} \dot{u}_{F} / c \\
1 / L & 0 & 0 \\
0 & 0 & 0
\end{array}\right] \\
{\left[u(0), i_{L}(0), z\right]=\left[\begin{array}{cc}
\left.0, g_{F}\left(u_{F 1 x}+u_{F 2 x}\right), 0\right], \quad[u(0), \dot{u}(0)]=[0,0] \\
\text { LE1 }=-2.49 \mathrm{e}+002, \quad \mathrm{LE} 2=-1.10 \mathrm{e}+006, \text { LE3 }=0.00176 \text { when } T_{\text {run }}=\text { step } * 10^{4}
\end{array}\right.} \\
\text { LE1 }=-1.79 \mathrm{e}+002, \text { LE2 }=-1.10 \mathrm{e}+006, \text { LE3 }=9.34 \mathrm{e}-006 \text { when } T_{\text {run }}=\text { step } * 10^{5}
\end{gathered}
$$

The operation of LE program takes initial value at $t=0$ as shown in (23). It can be found that the maximum Lyapunov Exponent LE3 is positive when the operation time $T_{\text {run }}=$ step $* 10^{4}$ is shorter as shown in (24). The phase portraits show a chaotic state as shown in Figure 2(a). While LE3 tends to zero when the operation time $T_{\text {run }}=$ step $* 10^{5}$ is longer, as shown in (25). The LE have two negative and one zero when running time is long enough, and a periodic state appears as shown in Figure 2(b). The phase portrait evolves and converges to the 


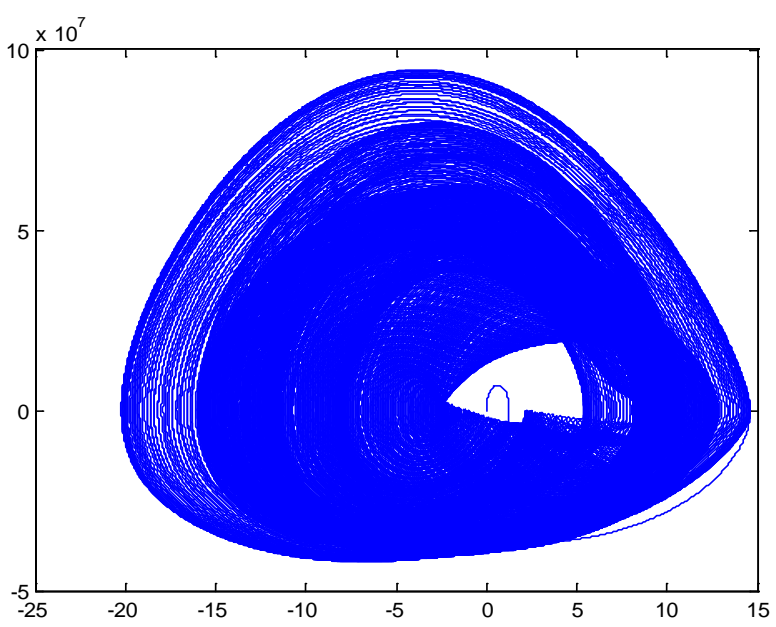

(a)

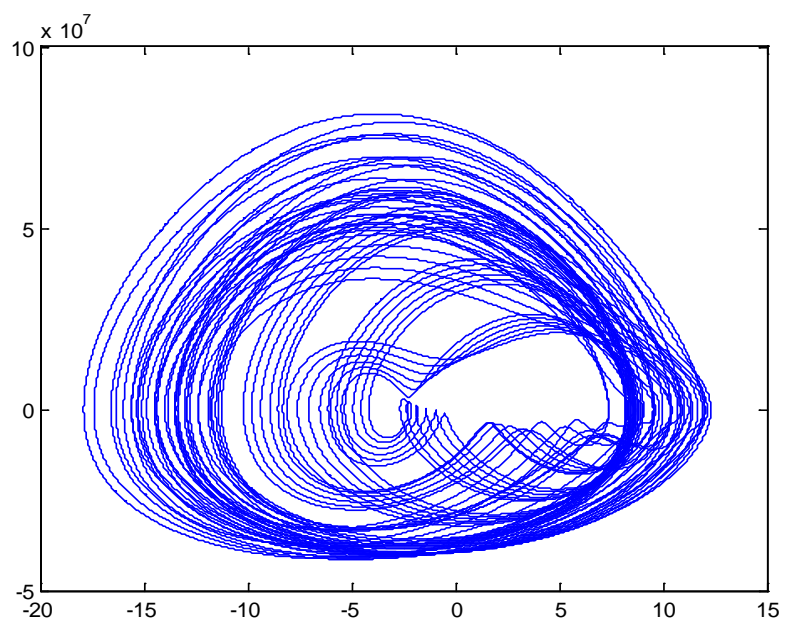

(b)

Figure 2. The $u-\dot{u}$ plane phase portrait of Table 1(e). (a) Drawing for $100 \%$ phase points; (b) Drawing for last $5 \%$ phase points.

final periodic state from chaotic state at the beginning.

There is only forced oscillation in this circuit entering steady state, and there is no freedom self-oscillation. Finally, freedom component inevitably exhausts to zero because there is only positive resistance element in the network. Excited source energy export to the third harmonic (difference frequency) through frequency conversion. These three harmonic components occupy predominant positions in the Fourier series expansion. The results from combined excitation of two signal sources are equivalent to a non-sinusoidal periodic source including two harmonic components $\omega_{1}$ and $\omega_{2}$. If calculation is strict and accurate, then common base frequency is $\omega_{\text {COM }}=0.01$, and period is $T=2 \pi / \omega_{\text {COM }}=628.32$ seconds (if MATLAB operation can achieve such precision). Various harmonic components are the integer multiples of $\omega_{\text {СOM }}$. When the simulation time $T_{S}<T$ or LE operation time $T_{\text {run }}<T$, phase portrait plotted hasn't finished one cycle, it is shown as aperiodic chaos.

If such high precision is not reached when the phase portrait is drawn by Matlab numerical simulation, the drawn phase portrait may become periodic state when $w_{1}, w_{2}$ mantissa 0.01 is ignored, and cycle is $T=6.2832$ second. Therefore, the precision of numerical simulation will also affect the character of phase portrait.

The oscillation behaviors for the overall output of mixing circuits can be divided into two kind of pattern of the periodic and chaotic state. However, the data handled by Matlab are all rational numbers, so the drawn phase portrait is all periodic track. In fact, there is only periodic function when the phase portrait is drawn by numerical simulation. The chaotic oscillation can be considered as sufficient or infinite extension of period $T$.

\subsection{Phase Portrait of Second Type Mixing Oscillation}

Taking the Table 2(a) as an example, the $u-\dot{u}$ plane phase portrait of overall output of mixing oscillation is drawn by Simulink, as shown in Figure 3. The Figure 3(a) takes 100\% phase points, and Figure 3(b) takes the last $1 / 1000$ phase points.

It is clear that distinguishing chaotic or periodic state cannot be directly perceived. The phase portraits of the mixing oscillation are either chaotic or periodic states. If their cycle lengths are moderate, neither very short nor very long, distinguishing strictly the oscillation behavior is difficult. The phase portrait characters are closely related to the simulation time. The phase portrait appears non-periodic when simulation time $T_{S}$ is shorter than one complete cycle $T$. The phase portrait shows periodic state when the simulation time $T_{S}$ is bigger than $T$. It can be seen from this example that the mixing circuits containing self-oscillation usually produce chaos, namely the $T_{S}$ smaller than $T$.

\subsection{Phase Portrait of Single Excitation}

Taking the Table 2(g) as an example, the $u-\dot{u}$ plane phase portrait of oscillation caused by single excitation is drawn by Simulink, as shown in Figure 4. The Figure 4(a) takes 100\% phase points, it contains transient 
process of the oscillation; and Figure 4(b) takes the last 1/1000 phase points, it only contains the process after entering steady state.

Non-linear oscillation refers to vibration with sustained oscillation character. It can be divided into two categories. One is periodic oscillation or constant periodic oscillation, in which dynamic variable tends to be steady with periodic repeatability. This kind of oscillation can be expressed as Fourier series. Another one is called non-constant periodic oscillation or aperiodic oscillation for short. Dynamic variable in such oscillation will keep oscillating continuously but without determination period. Although the orbit in phase portrait is without repetition, however the oscillation is sustained. Such a phenomenon is called aperiodic oscillation.

The example in Table 2(g), the oscillation can be divided into transient and steady state processes. The transient process belongs to aperiodic oscillation; the steady state process belong to periodic oscillation. For some nonlinear system, the aperiodic process can be extended infinitely or sufficiently. Within whole simulation time, aperiodic oscillation is unique state instead of temporary part of whole process, this is chaos. It is a very common bounded nonlinear function. The whole process of the oscillation cannot be divided into transient and stead-state.

It is another leap that human understands nature. The parasitic oscillation phenomenon, such as a mess on display had been found in nature a long time ago. It is difficult to distinguish that the mess are periodic or chaotic oscillations.

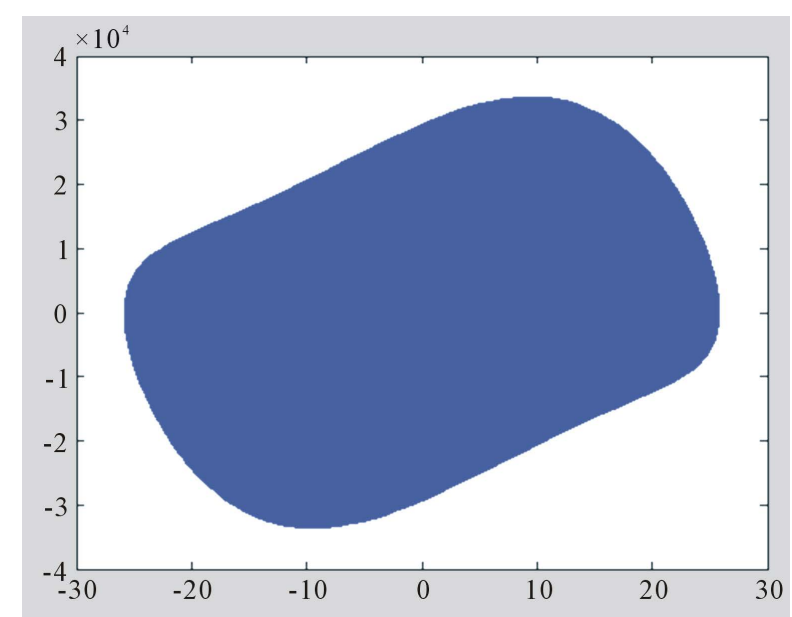

(a)

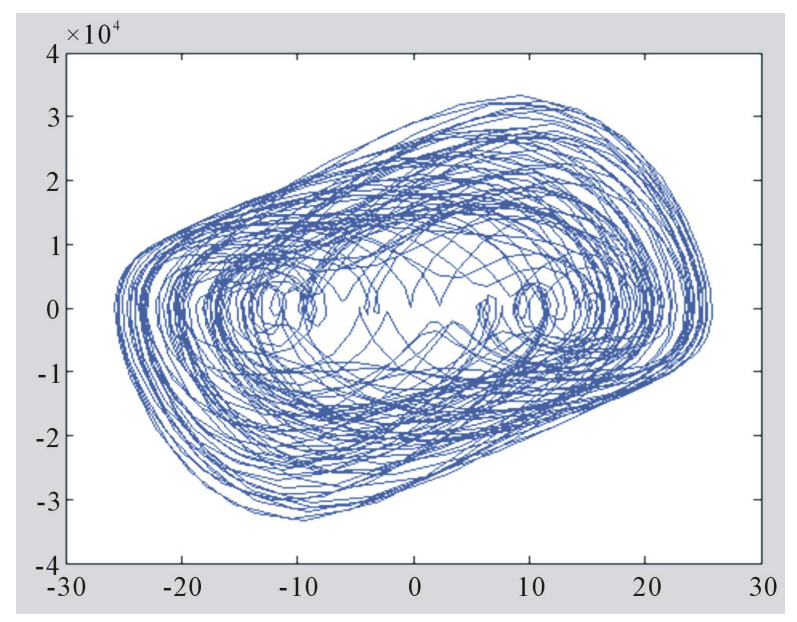

(b)

Figure 3. Phase portrait of Table 2(a). (a) Drawing for 100\% phase points; (b) Drawing for last 1/1000 phase points.

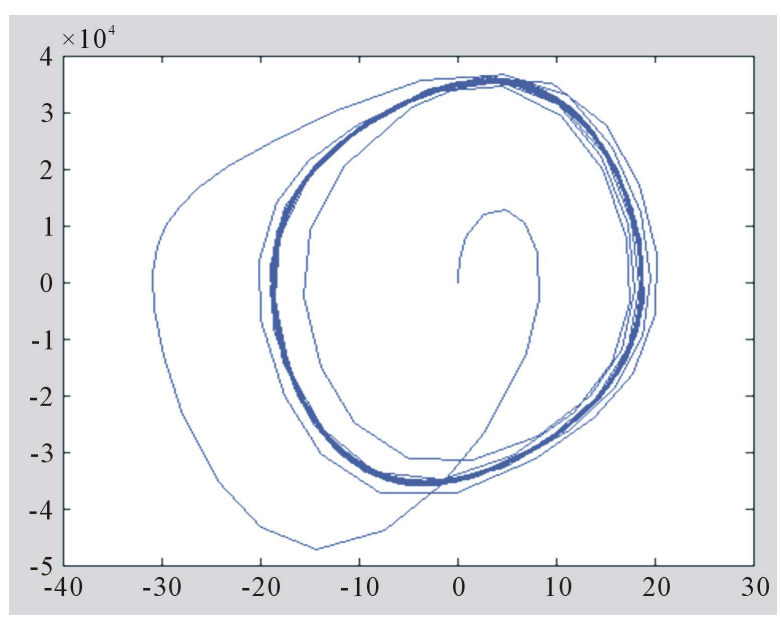

(a)

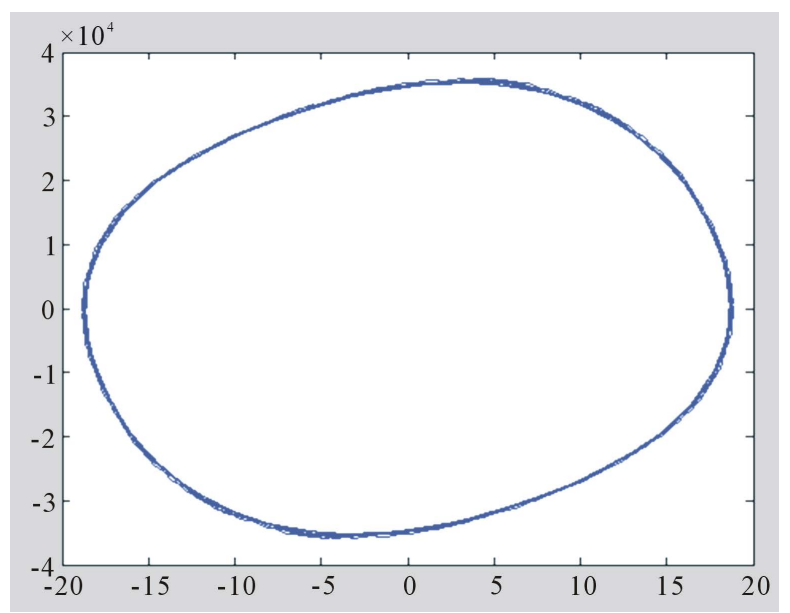

(b)

Figure 4. Phase portrait of Table 2(g). (a) Drawing for 100\% phase points; (b) Drawing for last 1/1000 phase points. 


\section{Conclusions}

1) In domestic and foreign literature, with regard to researching nonlinear circuits and chaos all are established on the basis of dynamic mechanics except the mathematic method of finding differential equations. The complex power balance theory opens up a new field for researching nonlinear oscillation. The correct harmonic components can be sought by power balance theorem of frequency domain. This paper propels the application of theorem to containing three main harmonics from single fundamental wave in the past.

The operation results of two kinds of programs (Tab1.nb and Power1.nb; uhp.nb and haveself.nb; onlyup.nb and nonself.nb) are consistent. It shows the pervasiveness of application of complex power balance theorem.

2) The chaos can be researched from the theory of frequency domain. This is an importance contribution of power balance theory. This paper propels the application of theory to chaotic oscillation. The main part of the steady-state oscillation solution is obtained through the power balance theory, its important contribution shows that the main harmonic solution is able to represent the basic part of both periodic solution and chaotic solution. Even though chaos is non-periodic within simulation time, its basic main part is periodic, and the main harmonic balance equations belong to linear equations.

3) The necessary and sufficient condition for periodic oscillation is that there should and must be only one unique common fundamental frequency. If there is no unique single common fundamental frequency, such as Chua's circuit, Lorenz system, Chen system, Lu system, Liu system, Qi system, etc., because their solutions cannot be expressed in Fourier series, they all belong to aperiodic chaotic oscillation.

If there are more than two main harmonic waves in the circuit, chaotic oscillation is possibly produced. The phase portraits in Table 2(a)-(c) show that chaos usually can be produced by the mixing of multi-harmonic. The more the harmonic components of involving mixing are, the greater the possibility of producing chaos is.

4) Steady-state oscillation solution is obtained through the harmonic-coefficient balance formulae of differential equation. Its roots may contain more than ten groups sometimes. A reasonable solution should be selected by power balance theorem. The complex power of every harmonic component totals zero. There is no self-excited oscillation if the amplitude meeting power balance requirements is an imaginary root $U_{h m}^{2}<0$. The (20) can be used to determine whether there is self-excited oscillation, and thus a very reliable result can be got. Its correctness can be verified with the phase portrait.

5) There are two types of harmonic components of non-excitation frequency in the network. One is the selfoscillation frequency. Its power is produced by the non-linear element $g_{N}$ with the characteristic of comprising negative term as shown in (11), and it can produce power by itself without excitation source. The greater the voltage amplitude is, the smaller the power produced by the $g_{N}$ of comprising negative term is. The power produced by the $g_{N}$ is insufficient to compensate the consumed power in positive conductance $g_{F}$ when amplitude increases up to a certain value, then self-excited oscillation disappears.

The other harmonic component is produced by variable frequency element of containing only positive characteristics as shown in (1), and it cannot produce power by itself without excitation source. It only plays transformation action. The power consumption of difference frequency component cannot be directly replenished from the power exported by excited source. It is necessary to convert excited power into the power of difference frequency component through frequency conversion element. The variable-frequency element is the source of difference-frequency power which is produced by its transformation instead of directly by itself. The difference frequency component of non-excited frequency always exists, when amplitude increases up to sufficiently large.

\section{Acknowledgements}

This work was supported by National Natural Science Foundation of China (60662001).

\section{References}

[1] Feng, J.C. and Li, G.M. (2012) Journal of South China University of Technology (Natural Science Edition), 40, 13-18.

[2] Huang, B.H., Huang, X.M. and Li, C.B. (2011) Mathematics in Practice and Theory, 41, 172-179.

[3] Huang, B.H., Niu, L.R., Lin, L.F. and Sun, C.M. (2007) Acta Electronica Sinica, 35, 1994-1998.

[4] Huang, B.H., Huang, X.M. and Wei, S.G. (2008) Journal on Communications, 29, 65-70.

[5] Huang, B.H., Chen, C., Wei, S.E. and Li, B. (2008) Research \& Progress of Solid State Electronics, 28, 57-62.

[6] Huang, B.H., Huang, X.M. and Wang, Q.H. (2006) Research \& Progress of Solid State Electronics, 26, 43-48. 
[7] Huang, B.H. and Kuang, Y.M. (2007) Journal of Guangxi University (Natural Science Edition), 32.

[8] Huang, B.H., Li, G.M. and Wei, Y.F. (2012) Modern Physics, 2, 60-69.

[9] Huang, B.H., Huang, X.M. and Li, H. (2011) Main Components of Harmonic Solutions. International Conference on Electric Information and Control Engineering, New York, 15-17 April 2001, 2307-2310.

[10] Huang, B.H., Huang, X.M. and Li, H. (2011) Procedia Engineering, 16, 325-332. http://dx.doi.org/10.1016/j.proeng.2011.08.1091

[11] Huang, B.H., Yang, G.S., Wei, Y.F. and Huang, Y. (2013) Applied Mechanics and Materials, 325-326, $1508-1514$.

[12] Huang, B.H., Li, G.M. and Liu, H.J. (2013) Modern Physics, 3, 1-8. http://dx.doi.org/10.12677/MP.2013.31001 
Scientific Research Publishing (SCIRP) is one of the largest Open Access journal publishers. It is currently publishing more than 200 open access, online, peer-reviewed journals covering a wide range of academic disciplines. SCIRP serves the worldwide academic communities and contributes to the progress and application of science with its publication.

Other selected journals from SCIRP are listed as below. Submit your manuscript to us via either submit@scirp.org or Online Submission Portal.
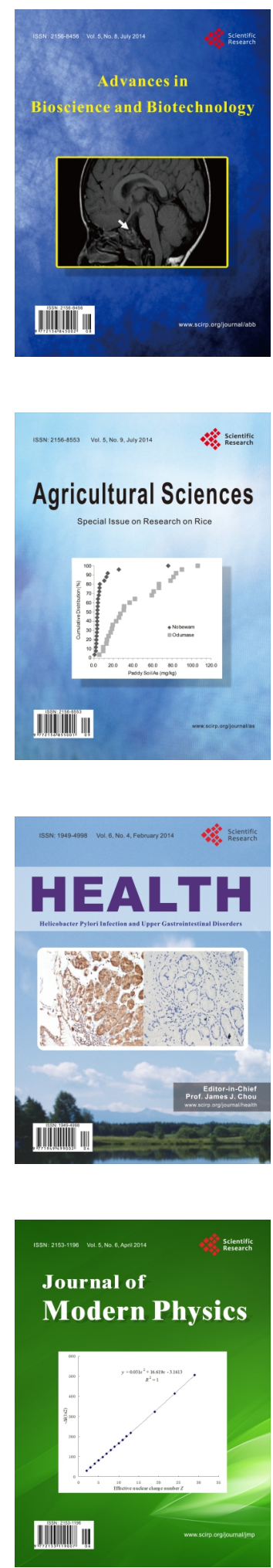
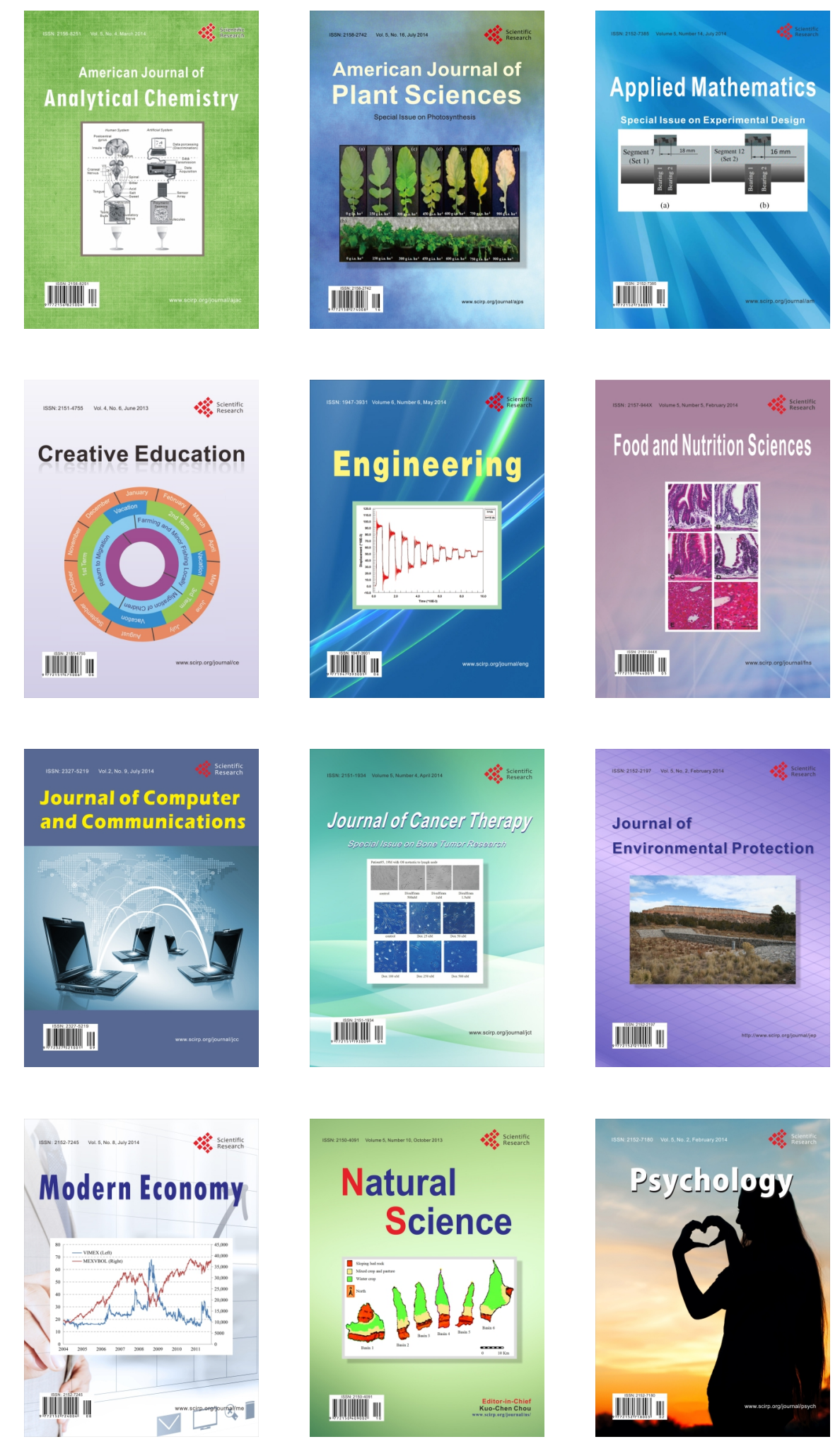